\title{
On the way to universal coverage of maternal services in Iringa rural District in Tanzania. Who is yet to be reached?
}

\author{
Manuela Straneo ${ }^{1}$, Piera Fogliati ${ }^{1}$, Ingrid Pellis ${ }^{1}$, Catherine Goodman ${ }^{2}$, Donata Dalla Riva ${ }^{1}$, \\ Firma Kisika $^{3}$, Ezekiel Mpuya ${ }^{4}$, Giovanni Putoto ${ }^{1}$
}

\section{Doctors with Africa- CUAMM, Tanzania.}

2. London School of Hygiene and Tropical Medicine.

3. Iringa District Council, Reproductive and Child Health, District Medical Office.

4. Regional Medical Office, Iringa Region.

\begin{abstract}
Background: Strategies to tackle maternal mortality in sub-Saharan Africa include expanding coverage of reproductive services. Even where high, more vulnerable women may not access services. No data is available on high coverage determinants. We investigated this in Tanzania in a predicted high utilization area.

Methods: Data was collected through a household survey of 464 women with a recent delivery. Primary outcomes were facility delivery and $\geq 4$ ANC visits. Determinants were analysed using multivariate regression.

Results: Almost all women had attended ANC, though only $58.3 \%$ had $\geq 4$ visits. $\geq 4$ visits were more likely in the youngest age group (OR 2.7 95\% CI 1.32-5.49, p=0.008), and in early ANC attenders (OR 3.2 95\% CI 2.04-4.90, p<0.001). Facility delivery was greater than expected (87.7\%), more likely in more educated women (OR $2.795 \%$ CI 1.50-4.75, p=0.002), in those within 5 kilometers of a facility (OR $3.295 \%$ CI 1.59-6.48, p=0.002), and for early ANC attenders (OR $2.495 \%$ CI 1.20-4.91, p=0.02). Conclusion: Rural contexts can achieve high facility delivery coverage. Based on our findings, strategies to reach women yet unserved should include promotion of early ANC start particularly for the less educated, and improvement of distant communities' access to facilities.
\end{abstract}

Keywords: Maternal health services, delivery/obstetric, utilization, prenatal care, Tanzania, Africa, south of the Sahara.

DOI: http://dx.doi.org/10.4314/ahs.v16i2.10

Cite as: Straneo M, Fogliati P, Pellis I, Goodman C, Riva DD, Kisika F, et al. On the way to universal coverage of maternal services in Iringa rural District in Tanzania. Who is yet to be reached? Afri Health Sci 2016;16(2): 420-428. http:/ / dx.doi.org/10.4314/ abs. $v 16 i 2.10$

\section{Background}

Maternal mortality reduction remains a public health concern. It is expressed in Sustainable Development Goal 3 (SDG 3), which also identifies universal reproductive health coverage as a target. There were 289,000 maternal deaths globally in 2013 alone ${ }^{1}$. Maternal mortality ratios (MMR) are highest in sub-Saharan Africa, and Tanzania is no exception. Despite a relatively well developed primary
Corresponding author:
Manuela Straneo,
Doctors with Africa-CUAMM, Tanzania
Email:m.straneo@cuamm.org,
gambettas@hotmail.com

health care network ${ }^{2}$, and substantial progress towards reducing under 5 mortality ${ }^{3}$, Tanzania's MMR remains high, estimated at 449 per 100000 live births ${ }^{4}$.

A key intervention to reduce maternal mortality is skilled attendance at birth (SBA), but in Tanzania this was achieved in only $47 \%$ of deliveries in 2004/5, dropping to $34.5 \%$ in rural areas ${ }^{5}$. Coverage is highly variable across the country.

Antenatal care (ANC) uptake is essential for universal reproductive coverage. Though not expected to have a direct impact on mortality, ANC represents women's entry point into health services, and an opportunity to increase $\mathrm{SBA}^{6}$. Tanzania has relatively high ANC coverage: over $95 \%$ of women have at least one visit, compared to $71 \%$ for sub-Saharan Africa ${ }^{3}$, and $61.5 \%$ receive four or more visits ${ }^{5}$. 
As maternal mortality reduction strategies take effect, a progressive increase in utilization of reproductive services is anticipated. What will happen in high coverage areas? A minority of women, possibly the most vulnerable, will still fail to access services. Determinants may change as coverage increases. Understanding them where coverage is high will allow to fine tune strategies, enabling it to reach those left out.

Literature from sub-Saharan Africa has highlighted the importance of economic and physical accessibility and perceived need in determining SBA coverage ${ }^{7}$, with prorich inequalities well documented ${ }^{8}$.

Rural, poor and less educated women in sub-Saharan Africa have been shown to be less likely to access care, and many women start ANC late ${ }^{8-10}$. ANC is in turn a determinant of SBA. In Tanzania, studies in areas of intermediate coverage have found SBA to be significantly associated with distance to facility in two studies, and with socio-economic status (SES) in one study, but not in others $^{6,11-13}$. No published studies have addressed quantitative determinants of ANC in Tanzania.

Moreover, no studies from sub-Saharan Africa have addressed SBA and ANC determinants in high coverage areas.

This study addresses this gap by analysing data from Iringa, Tanzania. The area has relatively high reproductive health services' coverage. The more recent TDHS findings support the hypothesis that Iringa District is a high coverage area for skilled birth attendance (facility delivery is often used as a proxy for $\mathrm{SBA}^{7}$; it has increased from $72 \%$ (TDHS 2004/5) $)^{5}$ to $80 \%$ (TDHS 2010) ${ }^{14}$ for the whole Iringa region. No disaggregated District data is available.

Results are presented from a survey carried out in Iringa rural District, which aimed to confirm high coverage of maternal services in this rural area and investigate determinants of use, to draw implications for meeting the needs of women who remain unserved.

\section{Methods}

The study was a cross-sectional household survey in Iringa District, one of eight districts of the then Iringa Region, in the Southern Highlands of Tanzania. It has a mostly rural population of approximately 260,000. Health facilities include the diocesan-owned district designated hos- pital, six health centres, and 59 dispensaries. Women can deliver in all levels of facilities. The district health system has a pyramidal structure, with dispensaries at the base. Each level refers complications to the one above, though services available in dispensaries and health centres generally do not differ. While the district hospital can offer all functions of comprehensive emergency obstetric care, none of the peripheral health facilities can be classed as basic emergency obstetric Care units. The District was part of a health system strengthening project implemented by a non-governmental organization from 2009-2012, which focused on access, quality and equity of reproductive health services.

A representative sample of the district population was obtained through two-stage cluster sampling. The sample size of 431 women (730 households) was based on estimating the primary indicator of facility delivery, using an expected prevalence of 75\% (TDHS 2010), sampling error of $5 \%$ and design effect of $1.5^{15}$. Thirty villages were randomly chosen in the first stage with probability proportional to size, based on population projections from the Tanzania 2002 census. Twenty-five households were selected in each village in the second stage through random systematic sampling.

A random walking direction was chosen by bottle spinning by the village office and consecutive households in the selected direction were visited. When inhabitants could not be located, the next household in the same direction was interviewed.

Data was collected in October 2009 by trained female interviewers using a pre-tested structured questionnaire. The survey was conducted with the permission and the full cooperation of the council health management team. All selected households were asked about household access to water, sanitation and socio-economic assets, and where available one woman of childbearing age (15-49 years) who had delivered in the previous five years was interviewed on her care seeking. If the household had $>1$ eligible woman present, one was selected using a table of random numbers and interviewed. Information on distance to the nearest health facility was collected from village authorities.

Data was double entered and cleaned in EpiData (version 3.1), and analysis was performed using STATA (version 
9.2). The STATA svyset command were used to specify survey design characteristics. Svy commands were used both in bivariate and multivariate analysis to account for clustered design.

The primary outcomes were ANC ( $\geq 4$ visits) and health facility delivery.

Socio-economic status (SES) was calculated based on household assets assessment ${ }^{16}$. Socio-economic scores were produced using durable household possessions, as applied by Bernard et $\mathrm{al}^{17}$ in rural Tanzania, a context very similar to that of the present study. Principal component analysis (PCA) was used to define weights for these as$\operatorname{sets}^{18}$. The score from the first component, which accounted for $41.5 \%$ of data variability, was used to construct five socio-economic quintiles.

Associations between the primary outcomes and potential explanatory variables (demographic characteristics,
SES, distance to health facility and timing and number of ANC visits received) were assessed using crude odds ratios. Variables which were significant in bivariate analysis or which were associated with the relevant outcome in previous studies were included in multivariate regression models to calculate adjusted odds ratios.

Ethical approval was obtained from the National Institute for Medical Research (Tanzania) and the London School of Hygiene and Tropical Medicine. All women provided written informed consent.

\section{Results}

The number of household visited was 919. Inhabitants were absent in 161 (17.5\%), mainly for work in distant fields or events such as funerals. Only 8 households $(0.9 \%)$ refused the interview. A woman with a delivery in the previous 5 years was present in 464 and all were interviewed. Characteristics of interviewed women are displayed in Table 1.

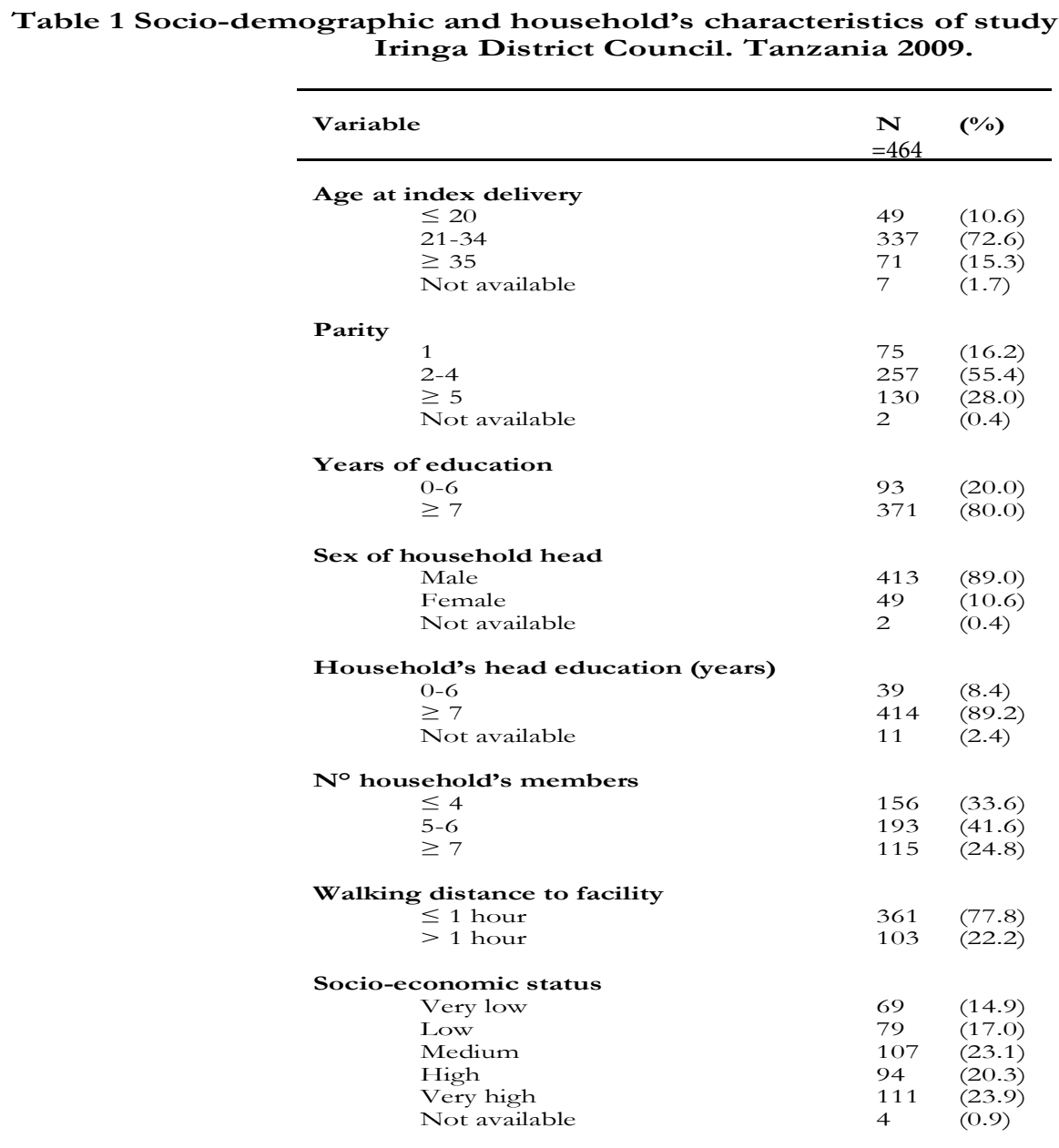


The majority belonged to Hehe and Bena tribes (80.6\%) and were subsistence farmers (82.7\%). Median age was 29 years (range 15-47), and median parity 3 (range 1-10). Mean age at first delivery was 20.0 years (19.6-23.3, 95\% $\mathrm{CI})$, and forty-one $(8.8 \%)$ were $<16$ at first delivery. Their households were mainly male-headed, with a mean size of 5.4 (5.2-5.6, 95\% CI). Over three quarters lived within one hour's walking distance to a facility.

Data on outcomes are available for 463 women. ANC coverage was almost universal with only 2 women reporting no attendance. Only 270 women $(58.3 \%$, 95\% CI 53.6-63.0) had $\geq 4$ ANC visits. One third (35.4\%) started ANC attendance in the first trimester as recommended (referred to as early ANC attendance).

Most women delivered in health facilities $(87.7 \%, 95 \%$ CI
83.4-92.0). Six (1.3\%, 95\% CI 0.3-2.3) delivered on the way and $51(11.0 \%, 95 \%$ CI 6.7-15.3) delivered at home.

Data on type of delivery is available for 454 women. Of these, $9.5 \%$ (95\% CI, 6.4-12.6\%) reported a cesarean section, the remaining a normal delivery.

Crude odds ratios showed that age at delivery and early ANC attendance were significantly associated with complete ANC (Table 2). Younger women (age $\leq 20$ years) were more likely to have $\geq 4$ ANC visits compared with older women $(p=0.005)$. Early ANC attendance was associated with a higher probability of completing 4 visits compared to later attendance $(\mathrm{p}<0.001)$.

After controlling for other variables and SES, younger age at delivery $(\mathrm{OR}=2.70,95 \% \mathrm{CI} 1.32-5.49)$ and early ANC attendance $(\mathrm{OR}=3.15,95 \% \mathrm{CI} 2.04-4.90)$ remained strongly associated with the outcome (Table 2).

Table 2. Factors associated with complete $(\geq 4)$ antenatal visits.

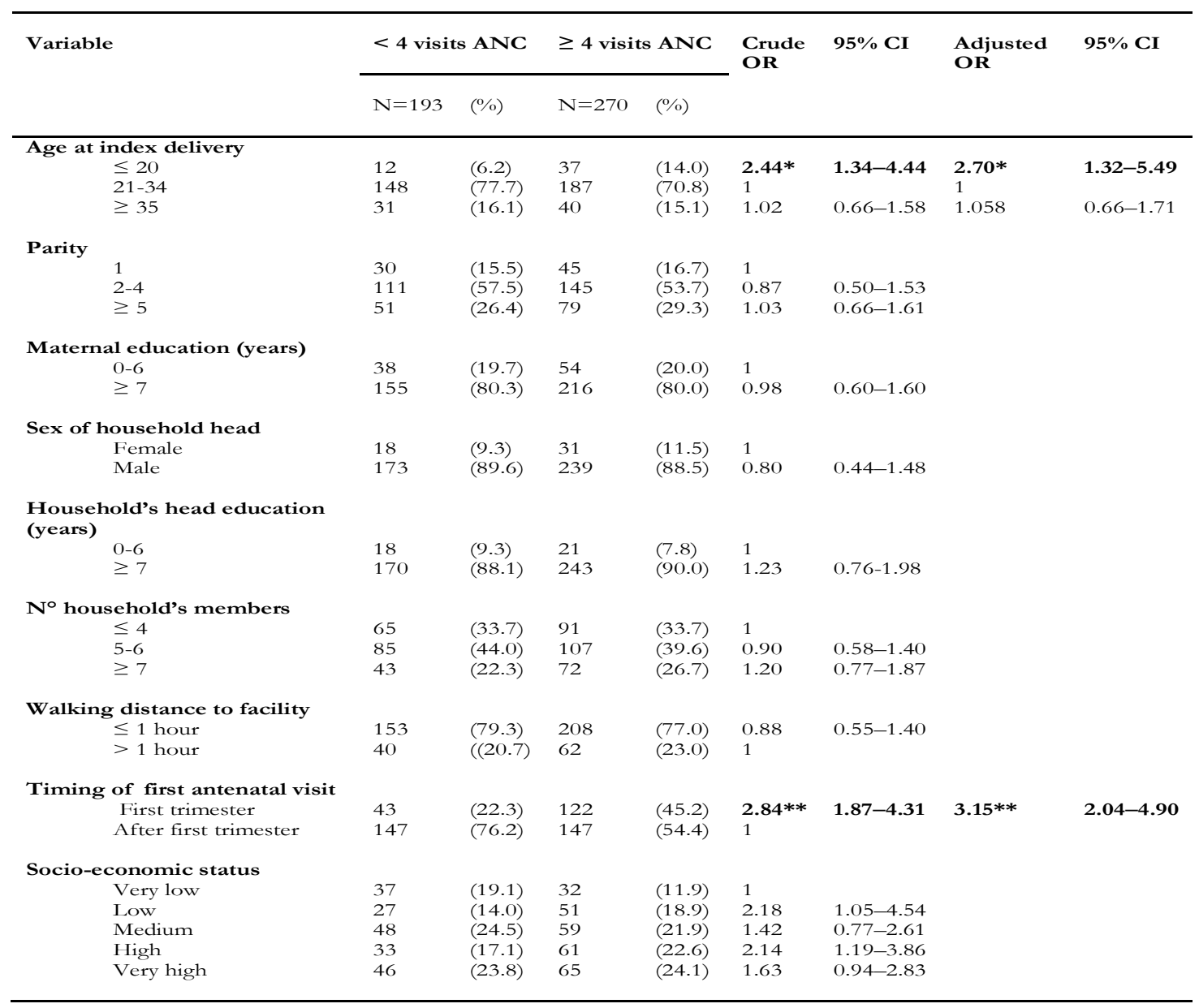

$* \mathrm{p}<0.05$
$* * \mathrm{p}<0.001$ 
Results on SES were inconsistent. There was some indication that the second lowest (OR 2.18, $\mathrm{p}=0.037)$ and second highest quantile (OR 2.14, $\mathrm{p}=0.013$ ) had significantly higher odds of complete ANC care, compared to the poorest group. There was some evidence $(p=0.033)$ that the two least poor groups pooled together, compared to the two poorest, had greater odds of $\geq 4$ visits (OR=1.77, 95\% CI 1.05-2.99, data not shown).

Health facility delivery was more likely for women with complete primary education or above ( $\geq 7$ years), compared to women with none/incomplete primary $<7$ years) $(p=0.001)$ (Table 3). There was some evidence of a linear trend indicating the odds of delivering at facilities improved with increasing years of education ( $\mathrm{p}$-value for linear trend $<0.001$, data not shown). All women with secondary education delivered in health facilities.
Distance to health facility was significantly associated with place of delivery. Women living within 1 hour walking distance were three times more likely to deliver in a facility compared to women living $>1$ hour away $(p=0.001)$. Here too there was evidence of a linear trend ( $\mathrm{p}$-value for linear trend $=0.0061$, data not shown).

Finally early ANC attendance, compared to late first attendance, was associated with health facility delivery $(p=0.02)$. There was no evidence of an effect of SES on the outcome.

Even after adjusting for significantly associated variables and SES, maternal education $(\mathrm{OR}=2.70,95 \% 1.58-4.80)$, distance to health facility (OR=3.21, 95\% CI 1.59-6.48) and early $\mathrm{ANC}$ attendance $(\mathrm{OR}=2.43,95 \% \mathrm{CI} 1.20-4.91)$ remained strongly associated with health facility delivery (Table 3).

Table 3. Factors associated with delivery at health facility.

\begin{tabular}{|c|c|c|c|c|c|c|c|c|}
\hline \multirow[t]{2}{*}{ Variable } & \multicolumn{2}{|c|}{$\begin{array}{l}\text { Delivery at } \\
\text { home or on the } \\
\text { way }\end{array}$} & \multicolumn{2}{|c|}{$\begin{array}{l}\text { Delivery at } \\
\text { facility }\end{array}$} & \multirow[t]{2}{*}{$\begin{array}{l}\text { Crude } \\
\text { OR }\end{array}$} & \multirow[t]{2}{*}{$95 \% \mathrm{CI}$} & \multirow[t]{2}{*}{$\begin{array}{l}\text { Adjusted } \\
\text { OR }\end{array}$} & \multirow[t]{2}{*}{$95 \% \mathrm{CI}$} \\
\hline & $\begin{array}{l}\mathrm{N}= \\
57\end{array}$ & $(\%)$ & $N=406$ & $(\%)$ & & & & \\
\hline \multicolumn{9}{|l|}{ Age at index delivery } \\
\hline$\leq 20$ & 4 & $(7.0)$ & 45 & $(11.1)$ & 1.53 & $0.39-5.98$ & & \\
\hline $21-34$ & 40 & $(70.2)$ & 295 & $(72.7)$ & 1 & & & \\
\hline$\geq 35$ & 12 & $(21.1)$ & 59 & $(14.5)$ & 0.67 & $0.35-1.27$ & & \\
\hline \multicolumn{9}{|l|}{ Parity } \\
\hline 1 & 5 & $(8.8)$ & 70 & $(17.2)$ & 1 & & & \\
\hline $2-4$ & 31 & $(54.4)$ & 225 & $(55.4)$ & 0.52 & $0.15-1.79$ & & \\
\hline$\geq 5$ & 21 & $(36.8)$ & 109 & $(26.9)$ & 0.37 & $0.11-1.26$ & & \\
\hline \multicolumn{9}{|l|}{ Maternal education (years) } \\
\hline $0-6$ & 21 & $(36.8)$ & 71 & $(17.5)$ & 1 & & & \\
\hline$\geq 7$ & 36 & $(63.2)$ & 335 & $(82.5)$ & $2.75 *$ & $1.58-4.80$ & $2.70 *$ & $1.50-4.75$ \\
\hline \multicolumn{9}{|l|}{ Sex of household head } \\
\hline Female & 4 & $(7.0)$ & 45 & $(11.1)$ & 1 & & & \\
\hline Male & 52 & $(91.2)$ & 360 & $(88.7)$ & 0.62 & $0.25-1.50$ & & \\
\hline \multicolumn{9}{|l|}{$\begin{array}{l}\text { Household's head education } \\
\text { (years) }\end{array}$} \\
\hline $0-6$ & 8 & $(14.0)$ & 30 & $(7.4)$ & 1 & & & \\
\hline$\geq 7$ & 47 & $(82.5)$ & 367 & $(90.4)$ & 2.08 & $0.94-4.63$ & & \\
\hline \multicolumn{9}{|l|}{$N^{\circ}$ household's members } \\
\hline$\leq 4$ & 17 & $(29.8)$ & 138 & $(34.0)$ & 1 & & & \\
\hline $5-6$ & 27 & $(47.4)$ & 166 & $(40.9)$ & 0.76 & $0.41-1.41$ & & \\
\hline$\geq 7$ & 13 & $(22.8)$ & 102 & $(25.1)$ & 0.97 & $0.49-1.90$ & & \\
\hline \multicolumn{9}{|l|}{ Walking distance to facility } \\
\hline$\leq 1$ hour & 32 & $(56.1)$ & 328 & $(80.8)$ & $3.29 *$ & $1.65-6.56$ & $3.21 *$ & $1.59-6.48$ \\
\hline$>1$ hour & 25 & $(43.9)$ & 78 & $(19.2)$ & 1 & & & \\
\hline \multicolumn{9}{|l|}{ Timing of first antenatal visit } \\
\hline First trimester & 12 & $(21.1)$ & 152 & $(37.4)$ & $2.17 *$ & $1.12-4.21$ & $2.43 *$ & $1.20-4.91$ \\
\hline After first trimester & 43 & $(75.4)$ & 251 & $(61.8)$ & 1 & & & \\
\hline \multicolumn{9}{|l|}{ ANC visits } \\
\hline Less than 4 & 25 & $(43.9)$ & 168 & $(41.4)$ & 1 & & & \\
\hline At least 4 or more & 31 & $(54.4)$ & 238 & $(58.6)$ & 1.14 & $0.64-2.04$ & & \\
\hline \multicolumn{9}{|l|}{ Socio-economic status } \\
\hline Very low & 10 & $(17.5)$ & 58 & $(14.3)$ & 1 & & & \\
\hline Low & 14 & $(24.6)$ & 65 & $(16.0)$ & 0.80 & $0.37-1.73$ & & \\
\hline Medium & 12 & $(21.1)$ & 95 & $(23.4)$ & 1.36 & $0.73-2.57$ & & \\
\hline High & 14 & $(24.6)$ & 80 & $(19.7)$ & 0.99 & $0.39-2.47$ & & \\
\hline Very high & 7 & $(12.3)$ & 104 & $(25.6)$ & 2.56 & $0.97-6.80$ & & \\
\hline
\end{tabular}




\section{Discussion}

A noteworthy finding which arises from the study is the remarkable coverage of institutional delivery: only one in ten women in Iringa District did not deliver in a health facility. It shows high coverage can be attained in rural sub-Saharan Africa. Though the finding does not equate into SBA as discussed below, it is a step on the way to reducing maternal mortality.

What makes this such a high coverage area for facility delivery? Differences in socioeconomic characteristics cannot easily explain this. $85 \%$ of the district population is rural, similar to other parts of Tanzania, and average income is well below the national poverty line ${ }^{19}$. Access of women to education does not appear greater than the rest of the country ${ }^{5}$. Ethnic mix may be partly responsible, as nomadic pastoralists, who generally choose home delivery, are rare in this district ${ }^{5}$. On the supply side, a high health facility density appears a likely determinant: in Iringa, the population per health facility is nearly half the National average (3939 people per health facility in Iringa district compared to 7786 in Tanzania overall). This is the result of early application of National policies to increase available health facilities (a dispensary in every village ${ }^{20}$ ), due to demand side factors: the geographical setting with highlands and lowlands implies particular access challenges for the rural population.

The sample size was not sufficient to estimate the MMR in this district so it is not possible to assess whether this high facility delivery coverage translated into fewer deaths, but a negative logarithmic relationship has been reported for SBA and MMR in developing countries ${ }^{5}$.

Looking at the other outcome, the study found almost all women had attended ANC at least once during their pregnancy, but less than two thirds of women had $\geq 4$ visits; coverage of $\geq 4$ ANC remains unsatisfactory.

Analysis of determinants of the two outcome variables helps to answer the question of who is yet unreached. Women who did not deliver in facilities were more likely to be less educated, live over one hour's walking distance from the facility, and to have started ANC after their first trimester. Early attendees may be more concerned about their pregnancy, thus attend ANC regularly and choose facility delivery. Those who failed to complete ANC were more likely to be over 20 years of age and to have started ANC after their first trimester. Higher OR for under 20s cannot easily be explained as all women are advised to undergo at least four visits during prenatal care; perhaps a perception of greater risk of this age group by midwives may lead to a stronger recommendation. SES was not clearly associated with either ANC or SBA; this may have reflected a lack of statistical power, though large effects are unlikely for SBA due to high coverage levels.

In an effort to improve maternal services to reach all women, the key factors worth underlying to policy makers are distance to health facility, education and early ANC attendance.

The findings indicate that communities distant from the nearest facility are disadvantaged with regards to delivery services, though not for complete ANC coverage. The discrepancy maybe explained by outreach clinics for ANC, carried out by health staff to villages with no health facility. This can partly offset distance barriers for antenatal care. To reach the women who are left out from childbirth services, strategies to improve distant communities' access must be sought. Physical accessibility is particularly challenging in rural settings, and lack of transportation is the primary preventable cause of death ${ }^{5}$. In Tanzania, the use of simple motorized tricycle emergency obstetric transport (bajaj ambulances) is under evaluation in some rural areas. From existing literature, evidence is available on the effectiveness of voucher schemes, which could be used for to cover transport $\operatorname{costs}^{21}$. Promotion of maternity waiting homes is also advocated ${ }^{22}$, and the use of mobile phones has potential to improve communication for distant communities ${ }^{23}$.

Less educated women also appear left out from high delivery services coverage. Though education levels cannot be addressed by health planners, this should be considered in health promotion efforts, utilizing means appropriate for this more vulnerable group (discussed below). The third determinant identified is late start of antenatal care. This is important to identify women who are left out from both outcomes. Regarding complete ANC coverage, which remains challenging in this setting, existing evidence indicates community involvement can be effective in improving access to early ANC care ${ }^{24}$, and it could therefore be intensified in these target groups to improve both ANC and facility delivery. Community health workers are an asset at village level in Tanzania which can be exploited to reach women with lower education. Health promotion programs must use means appropriate for 
this target group, such as theatre for health education and non-written tools for information, education and communication activities.

High uptake of obstetric services gives the District huge potential to achieve universal reproductive coverage. However, as maternal mortality is inversely related to skilled attendance at birth, rather than to facility delivery, gaps in human resources must be addressed. In $2010^{19}$, total health staff shortage in the district was $48.8 \%$, with 312 positions out of 639 unfilled, and Dispensaries are at times staffed only by under-qualified providers. National policies to improve this grim picture are necessary to ensure that health facility delivery does in practice equate to SBA for all women.

Some limitations should be considered in discussing these findings. First, as in other published work ${ }^{7,12}$, SBA has been approximated to health facility delivery, yet some facilities lack staff who can provide $\mathrm{SBA}^{2}$. Asking women about their attendant could have addressed this, but there is evidence of inaccurate identification of attendants' skill level by women ${ }^{25}$. Moreover, Kenney et al. ${ }^{3}$ underline that "to save most lives, coverage of care alone is not enough", and quality of services must be ensured, meaning that any gap in skills, equipment, transportation must also be addressed ${ }^{20}$.

Secondly, the sampling technique for households may have introduced selection bias, due to the greater likelihood of interviewing households near the village centre given the starting point of the village office. In other studies in similar settings, vitongoji (village subunits) rather than villages were randomly selected in the first stage $^{12,13}$ which would have reduced the tendency to select households from the village centre. However, in this context there is no evidence of a systematic distribution of dwellings within villages.

Thirdly, the possibility of poor recall must be considered, as the index pregnancy could have been up to five years previously. For both health facility delivery and ANC, social desirability bias may have led respondents to over-report use of formal facilities. However, though validation of women's responses was not possible, as ANC cards are retained by health facilities after delivery and women receive no discharge forms after delivery, triangulation with routinely collected data both at district level and National level ${ }^{14,19}$ supports the study's findings, indicating recall/ social desirability did not play a relevant role.

Distance to health facility was collected at village level; in larger communities distance may have varied substantially within the village meaning that some households could have been mis-classified. Finally no information was collected on perceived need/benefit of maternal health services $^{26}$, thus their effects on access could not be assessed.

\section{Conclusion}

The survey offers a new perspective on maternal services for the rural poor. Excellent coverage of delivery services can be achieved in rural areas. Complete ANC coverage remains a challenge in this setting, emphasising the continued importance of policies to improve its uptake.

Women who are left out in this context include women whose home is $>5 \mathrm{~km}$ from a health facility, women with no or incomplete education, and women who do not start ANC early. Strategies to further increase coverage of reproductive health services and thus reduce MMR should include promotion of early ANC through community involvement, particularly for less educated women, and improvement of distant communities' access to health facilities.

\section{Acknowledgements}

Collaboration with Iringa District Council and the District Medical Office for this study was greatly appreciated. The contribution of the women interviewed from Iringa District, who hopefully will benefit from this work, made the study possible.

Sam Pattenden, LSHTM, kindly provided statistical support.

The survey was funded by the Italian Cooperation, as part of a Doctors with Africa-CUAMM development project.

\section{References}

1. World Health Organization. Trends in maternal mortality: 1990 to 2013. Estimates by WHO, UNICEF, UNFPA, The World Bank and the United Nations Population Division. Geneva, WH0;2014. ISBN 9789241503631

2. Kruk ME, Mbaruku G, McCord CW, Moran M, Rockers PC, Galea S. Bypassing primary care facilities for childbirth: a population-based study in rural Tanzania. Health Policy and Planning. 2009; 24 (4): 279-288. http:/ /dx.doi. org/10.1093/heapol/czp011 
3. Kinney MV, Kerber KJ, Black RE, et al. Sub-Saharan Africa's Mothers, Newborns, and Children: Where and Why Do They Die? PLoS Med 2010; 7 (6): e1000294. http://dx.doi.org/10.1371/journal.pmed.1000294

4. Hogan MC, Foreman KJ, Naghavi M, et al. Maternal mortality for 181 countries, 1980-2008: a systematic analysis of progress towards Millennium Development Goal 5. The Lancet. 2010; 375(9726): 1609 -1623. http:/ / dx.doi.org/10.1016/S0140-6736(10)60518-1

5. National Bureau of Statistics [Tanzania] and ORC Macro. Tanzania Demographic and Health Survey 20042005. Dar es Salaam, Tanzania: National Bureau of Statistics and ORC Macro. 2005 [cited 2009 Jun 16]. Available from: http://www.nbs.go.tz/DHS/index.htm

6. von Both C, Flebetaa S, Makuwani A, Mpembeni R, Jahn A. How much time do health services spend on antenatal care? Implications for the introduction of the focused antenatal care model in Tanzania. BMC Pregnancy and Childbirth. 2006; 6(1): 22-30. http://dx.doi. org/10.1186/1471-2393-6-22

7. Gabrysch S, Campbell OMR. Still too far to walk: literature review of the determinants of delivery service use. BMC pregnancy and childbirth. 2009; 9: 34-52. http:// dx.doi.org/10.1186/1471-2393-9-34

8. Houweling TAJ, Ronsmans C, Campbell OMR, Kunst AE. Huge poor-rich inequalities in maternity care: an international comparative study of maternity and child care in developing countries. Bulletin of the World Health Organization. 2007; 85(10): 745-754. http://dx.doi. org/10.2471/BLT.06.038588

9. Simkhada B, Teijlingen ER van, Porter M, Simkhada P. Factors affecting the utilization of antenatal care in developing countries: systematic review of the literature. Journal of Advanced Nursing. 2008; 61(3): 244-260. http:// dx.doi.org/10.1111/j.1365-2648.2007.04532.x

10. World Health Organization. Antenatal care in developing countries. Promises, achievements and missed opportunities: an analysis of trends, levels and differentials. 2003 [cited 2011 Sep 20];36. Available from: http:/ /www. who.int/reproductivehealth/publications/maternal_ perinatal_healt h/9241590947/en/index.html

11. Mpembeni R, Killewo J, Leshabari M, et al. Use pattern of maternal health services and determinants of skilled care during delivery in Southern Tanzania: implications for achievement of MDG-5 targets. BMC pregnancy and childbirth. 2007; 7(1): 29. http://dx.doi. org/10.1186/1471-2393-7-29

12. .Mrisho M, Schellenberg JA, Mushi AK, et al. Factors affecting home delivery in rural Tanzania. Tropical Medicine \& International Health. 2007; 12(7): 862-872. http:// dx.doi.org/10.1186/1471-2393-9-10

13. Rockers P, Wilson M, Mbaruku G, Kruk ME. Source of Antenatal Care Influences Facility Delivery in Rural Tanzania: A Population-Based Study. Maternal and Child Health Journal. 2009; 13(6): 879-885. http://dx.doi. org/10.1007/s10995-008-0412-

14. National Bureau of Statistics, Tanzania, ICF Macro Calverton, Maryland USA. Tanzanian Demographic and Health Survey 2010. Preliminary report. Dar es Salaam, Tanzania: 2010 [cited 2010 Nov 24]. Available from: www.nbs.go.tz

15. Minassian DC. J Comm Eye Health. 1997;10(23):42 -44 16. Filmer D, Pritchett LH. Estimating wealth effects without expenditure data-or tears: an application to educational enrolment in states of India. Demography. 2001; 38(1): 115 -132. http://dx.doi.org/10.1353/ dem.2001.0003

17. Bernard J, Mtove G, Mandike R, Mtei F, Maxwell C, Reyburn H. Equity and coverage of insecticide-treated bed nets in an area of intense transmission of Plasmodium falciparum in Tanzania. Malar J. 2009; 8:65. http:// dx.doi.org/10.1186/1475-2875-8-65

18. Vyas, S. \& Kumaranayake, L. Constructing socio-economic status indices: how to use principal components analysis. Health Policy and Planning. 2006; 21(6), pp.459. http://dx.doi.org/10.1093/heapol/czl029

19. District Planning Team. Iringa District Council Health Department - Comprehensive Council Health Plan for 2009/2010. 2009; Iringa Tanzania

20. Ministry of Health and Social Welfare, Tanzania. The National Road map Strategic plan to Accelerate reduction of Maternal Newborn and Child Deaths in Tanzania 2008-2015. United Republic of Tanzania. 2008. Available from: http://www.hdptz.esealtd.com/fileadmin/documents/.../One_MNCH_plan.pdf

21. Bellows NM, Bellows BW, Warren C. The use of vouchers for reproductive health services in developing countries: systematic review. Tropical Medicine and International Health. 2011; 16(1): 84-96. http://dx.doi. org/10.1111/j.1365-3156.2010.02667.x

22. Magoma M, Requejo J, Campbell OMR, Cousens S, Filippi V. High ANC coverage and low skilled attendance in a rural Tanzanian district: a case for implementing a birth plan intervention. BMC Pregnancy and Childbirth. 2010; 10(1): 13. http://dx.doi.org/10.1111/j.13653156.2010.02667.x 
23. Adegoke AA, van den Broek N. Skilled birth attendance-lessons learnt. BJOG. 2009; 116 Suppl :33-40. http://dx.doi.org/10.1111/j.1471-0528.2009.02336.x 24. Mushi D, Mpembeni R, Jahn A. Effectiveness of community based safe motherhood promoters in improving the utilization of obstetric care. The case of Mtwara Rural District in Tanzania. BMC pregnancy and childbirth. 2010; 10(1): 14. http://dx.doi.org/10.1111/j.14710528.2009.02336.x
25. Hussein J, Hundley V, Bell J, Abbey M, Asare CQ, Graham W. How do women identify health professionals at birth in Ghana? Midwifery. 2005; 21(1): 36 -43. http:// dx.doi.org/10.1016/j.midw.2004.09.009

26. Masanja H, de Savigny D, Smithson P, et al. Child survival gains in Tanzania: analysis of data from demographic and health surveys. The Lancet. 2008; 371: 1276-1283 . http:/ /dx.doi.org/10.1016/S0140-6736(08)60562-0 